\title{
LE METABOLISME DE L'EAU ET DES ELECTROLYTES EN NEUROLOGIE ET EN NEUROCHIRURGIE
}

\author{
ANDRÉ SOETENS, M.D. *
}

DePUIS PLUSIEURS ANNÉEs déjà de nombreux travaux, basés tant sur des observations cliniques que sur des recherches expérimentales, ont été consacrés à l'étude du métabolisme de l'eau et des électrolytes en rapport avec les affections neurologiques, neurochirurgicales et neuropsychiatriques.

L'intérêt pour cette étude trouve son origine dans quatre constatations importantes:

1) Les variations du métabolisme de l'eau dans certains syndromes psychiatriques, dans l'épilepsie et dans la paralysie périodique, où l'on constate en outre des fluctuations de la kaliémie.

2) Le syndrome d'hyperosmolarité que l'on constate dans certaines lésions traumatiques de la partie antérieure de la base du cerveau.

3) Le problème neurologique et neurochirurgical que constitue l'œdème cérébral, où l'on note, au niveau du tissu cérébral, une augmentation de la teneur en eau et une augmentation de la teneur en ion sodium.

4) Les troubles du métabolisme de l'eau et des électrolytes survenant au cours des suites opératoires.

Mon propos est de tenter de faire le tour du problème de la façon suivante: nous commencerons par un rappel de physiologie, qui comprendra quelques notes sur la physiologie cellulaire, le point actuel dans la discussion sur l'existence ou non d'un espace extracellulaire cérébral, ainsi que l'aspect endocrinologique de la régulation hydro-électrolytique. Nous envisagerons ensuite les manifestations cliniques consécutives au dérèglement de ce métabolisme, pour terminer par la proposition d'un schéma thérapeutique.

\section{RAPPEL DE PHYSIOLOGIE}

Les études de physiologie de ces dernières années nous permettent de comprendre comment des anomalies de fonctionnement peuvent engendrer un déséquilibre dans le métabolisme de l'eau et des électrolytes. Ces anomalies peuvent se localiser au niveau de la cellule nerveuse, au niveau de l'espace extracellulaire cérébral ou encore être la conséquence de facteurs endocriniens plus généraux.

\section{Physiologie cellulaire}

Dans ce domaine notre intérêt principal va vers la physiologie de la répartition ionique: celle-ci constitue essentiellement un problème de transport et de perméabilité membranaire.

\footnotetext{
"Service de Neurochirurgie Institut Bunge, Berchem-Anvers, Bclgique, et Département de Neurochimie Fondation Born-Bunge, Berchem-Anvers, Belgique.
} 
Grundfest ${ }^{1}$ et Hodgkin, ${ }^{2}$ étudiant l'axoplasme de l'axone géant du calmar, concluent que le potentiel de membrane, c'est-à-dire le potentiel de la cellule au repos, est réalisé par un potentiel électro-chimique déterminé par le gradient de concentration du potassium, qui est présent en forte concentration dans la cellule et en faible concentration en dehors de la cellule. Le potentiel d'action, par contre, est établi par le gradient de concentration du sodium, fort en dehors de la cellule et faible dans la cellule.

A l'état de repos la fibre nerveuse est plus perméable au potassium, alors que pendant le passage de l'influx nerveux il y a une augmentation transitoire mais très importante de la perméabilité au sodium ainsi qu'une diminution concommitante de la perméabilité au potassium, si bien que le gradient de concentration du sodium détermine le potentiel enregistré.

Hodgkin, reprenant ces expériences au moyen d'ions radioactifs, ${ }^{3}$ a produit la preuve que le maintien de l'inégalité de concentration des électrolytes est assuré par la membrane cellulaire agissant comme "pompe à sodium."

En 1957, Eccles a fait des mesures du potentiel de membrane et du potentiel d'action des neurones du système nerveux central par enregistrement au moyen de microélectrodes introduites dans les neurones moteurs et dans les cellules de Betz du chat et a constaté que le potentiel d'action est en général supérieur de 5 à $20 \mathrm{mv}$. au potentiel de membrane. ${ }^{4}$

Toutefois le passage d'ions de l'espace extracellulaire vers l'espace intracellulaire ou inversément suppose l'existence de modes de transport. Deux modes de transport se présentent: la diffusion passive et le transport actif.

Dans la diffusion passive on assiste à un mouvement de molécules d'une zône à haute concentration vers une zône à faible concentration. L'énergie nécessaire à ce mouvement est fournie par l'énergie thermique intrinsèque des molécules intéressées. Comme ce mouvement de molécules se fait à travers la membrane cellulaire, un facteur important dans la détermination du genre de substance qui sera ainsi transporté par diffusion passive est la composition de cette membrane cellulaire elle-même. D'après Fernandez-Moran ${ }^{5}$ la membrane cellulaire est constituée par une couche lipoïde bimoléculaire tapissée sur sa face interne et sur sa face externe d'une couche adsorbée de protéines.

Pour pouvoir pénétrer à travers la membrane, les substances chimiques doivent se trouver en solution. Il en résultera que c'est le degré de solubilité dans les lipides qui déterminera la facilité de pénétration de ces substances. Ce fait est à la base de la loi de Meyer-Overton qui rapporte l'efficacité des agents anesthésiques à leur degré de solubilité dans les lipides. Or, les ions étant non pas liposolubles, mais bien hydrosolubles, ce n'est pas ce mode de transport qui sera sollicité pour le passage d'ions à travers la membrane cellulaire.

Le transport actif, par contre, est le mouvement d'une substance à l'encontre d'un gradient électrochimique défavorable ou dans le sens d'un gradient favorable mais à une vitesse non compatible avec la seule diffusion thermique. Pour de tels mouvements une libération d'énergie est nécessaire: elle est fournie par des processus métaboliques, qui apparemment résident dans la membrane cellulaire elle-même. Dans cette membrane cette énergie serait fournie par divers substrats, enzymes et autres sources d'énergie. C'est par transport actif que se fait la régulation en sodium et en potassium. 
En 1950, Kety a démontré que le cerveau prend environ 25 pour cent de la consommation totale corporelle en oxygène, alors qu'il ne représente que 2 pour cent de la masse corporelle totale. ${ }^{6}$ Ce besoin important en oxygène se justifie par le transport constant d'ions à travers la membrane cellulaire au cours de l'activité cellulaire. Cette consommation considérable en oxygène se maintient pendant le sommeil et n'est pas modifiée par une activité mentale accrue..$^{7,8}$

Ainsi donc le protoplasme de chaque cellule se trouve enveloppé par une membrane semi-perméable, qui constituera, avec un éventuel espace extra-cellulaire, ce que l'on appelle la barrière hémato-encéphalique. Il est possible, ainsi que nous le verrons plus loin, que cet espace extra-cellulaire ne soit autre chose qu'un espace glial, c’est-à-dire une espace occupé par des prolongements des cellules gliales.

La barrière hémato-encéphalique est donc l'ensemble des structures qui séparent les milieux extérieurs (sang et liquide céphalo-rachidien) de la cellule nerveuse. C'est à travers cette barrière hémato-encéphalique que s'opèreront les échanges indispensables au métabolisme des cellules nerveuses.

La preuve de l'existence de cette barrière hémato-encéphalique a été apportée par de nombreux chercheurs: en 1928, Schaltenbrand et Bailey avaient déjà remarqué que l'injection de $\mathrm{NaCl}$ hypertonique produit une déshydratation marquée et prolongée du cerveau, laissant supposer que l'augmentation en concentration du $\mathrm{NaCl}$ plasmatique n'est pas suivie d'une augmentation correspondante de la teneur en $\mathrm{NaCl}$ du cerveau. ${ }^{9}$ Remontant plus loin nous voyons qu'en 1885 Ehrlich découvrit que la céruline A injectée par voie intraveineuse colorait tous les tissus sauf le tissu cérébral.

Cette barrière hémato-encéphalique reste cependant une structure controversée. Son existence pose le problème de l'espace extracellulaire que nous voulons discuter maintenant.

\section{Espace extra-cellulaire cérébral}

Robin, dans un article récapitulatif paru dans la revue "Anesthesiology" en $1965,^{10}$ a bien représenté l'évolution des découvertes et des théories au cours de ces dernières années. En effet, le concept de base d'espace extracellulaire s'établit sur trois hypothèses:

1) Il y a dans les tissus une quantité substantielle de liquide situé en dehors des cellules.

2) Une partie de ce liquide se présente comme un ultrafiltrat ou un dialysat du plasma: c'est le liquide interstitiel.

3) Ce liquide est au contact de la membrane cellulaire et sert d'intermédiaire dans les échanges entre les milieux intracellulaire et extracellulaire.

Classiquement la mesure de cet espace extracellulaire est basé sur l'utilisation de substances qui traversent librement la paroi vasculaire et se dispersent de façon homogène dans l'espace extracellulaire. Ces substances ne doivent pas pouvoir traverser la membrane cellulaire. Se basant sur le degré de diffusion de ces substances on peut calculer le volume de l'espace extracellulaire.

Deux types de substances ont été utilisés pour ce genre de mesure: 1) des hydrates de carbone, inertes au point de vue métabolique, tels que l'inuline (dont le poids moléculaire est de 5.000 ), qui ne pénètrent pas dans les cellules, 
probablement à cause de leur poids moléculaire; 2 ) des anions tels que $\mathrm{SO}_{4}{ }^{-}$et $\mathrm{Cl}^{-}$, qui ne pénètrent pas dans les cellules probablement à cause d'un gradient électrochimique défavorable.

Ces différentes substances mesurent toutefois des espaces différents. Cela explique que les auteurs obtiennent des valeurs qui ne scnt pas toujours concordantes. En se basant sur ces méthodes on a trouvé pour le tissu musculaire, par exemple, un espace extracellulaire d'environ 20 pour cent.

Des mesures effectuées par Reed et Woodbury, ${ }^{11}$ par Barlow et coll. ${ }^{12}$ et par Kibler et coll., ${ }^{13}$ au moyen d'injections intravasculaires d'inuline et d'ion $\mathrm{SO}_{4}^{-}$, ont démontré l'existence d'un espace extracellulaire cérébral d'environ 5 pour cent.

Par contre, des mesures basées sur le dosage de l'ion $\mathrm{Cl}^{-13}$ ont donné des valeurs de l'ordre de 35 pour cent. Il parait inconcevable que l'espace extracellulaire cérébral puisse avoir cette importance. Or de récentes études de ces mêmes auteurs ont établi qu'une partie importante du $\mathrm{CI}-$ cérébral se trouve dans les cellules: il s'avère done que l'on ne peut accepter les chiffres basés sur l'utilisation du $\mathrm{Cl}^{-}$endogène.

Afin d'éviter la barrière hémato-encéphalique, Rall et Patlak ${ }^{14}$ ont injecté de l'inuline dans l'espace subarachnoïdien et ont trouvé un espace extracellulaire d'environ 10 pour cent. En réalité cette étude a calculé le volume de liquide céphalo-rachidien présent dans le cerveau.

Au cours du Symposium sur la barrière hémato-encéphalique qui s'est tenu à Hilversum en 1966, ${ }^{15}$ Tower, Elliott et Pappius ont rediscuté les caractères biochimiques de l'espace extracellulaire et ont démontré que l'existence de cet espace est en effet confirmé par les méthodes biochimiques, mais que les résultats obtenus sont variables selon la technique mise en œuvre.

La question de savoir si le liquide céphalo-rachidien est le liquide extracellulaire fonctionnel du cerveau est restée sans réponse jusqu'ici. Toutefois deux caractéristiques du liquide extracellulaire dans les tissus périphériques ne peuvent être appliquées au liquide céphalo-rachidien. En effet:

1) Le liquide extracellulaire est un dialysat du plasma. Or la composition du liquide céphalo-rachidien ne peut s'expliquer comme dialysat du plasma: en effet la concentration en $\mathrm{Na}^{+}$, en $\mathrm{Cl}^{-}$et en $\mathrm{Mg}^{+}$du liquide céphalo-rachidien ne peut être la conséquence d'une simple diffusion. ${ }^{16}$ Cela implique donc que la composition du liquide céphalo-rachidien est règlée par l'activité métabolique des cellules responsables de la formation du liquide céphalo-rachidien.

2) Le liquide extracellulaire est un moyen direct d'échange entre cellules. Par contre, il est peu probable, à juger sur l'aspect anatomique, que le liquide céphalo-rachidien puisse remplir cette fonction d'échange tout au moins pour les cellules cérébrales plus profondes qui ne sont pas baignées par le liquide céphalo-rachidien, quoique certains auteurs, comme Fleischhauer, ${ }^{17}$ aient montré que certaines substances peuvent pénétrer entre les cellules nerveuses et ainsi quitter le liquide céphalo-rachidien.

Avant les biochimistes, les anatomistes s'étaient penchés sur le problème de l'existence d'un espace extracellulaire. Le microscope classique montrait de larges espaces entourant les éléments neuronaux: ces larges espaces furent considérés comme la contrepartie anatomique d'un espace extracellulaire fonctionnel.

Actuellement toutefois de nombreuses études au microscope électronique ${ }^{18-21}$ 
ont démontré qu'il n'y a pas d'espace extracellulaire important dans le tissu cérébral: les espaces entre les neurones sont occupés dans leur presque totalité par des cellules gliales. L'espace entre les membranes cellulaires est d'environ 100 à 150 Angstroms et est occupé par une substance amorphe à faible densité ressemblant à un gel.

Mais Lumsden ${ }^{22}$ et De Robertis et Gerschenfeld ${ }^{23}$ ont montré qu'une autre structure pouvait servir d'équivalent à l'espace extracellulaire dans le cerveau: c'est la masse des cellules gliales. Plusieurs faits démontrent que les cellules gliales constituent l'espace extracellulaire fonctionnel du cerveau:

1) Le microscope électronique montre que les cellules gliales occupent une position privilégiée par rapport aux capillaires cérébraux. De même les cellules gliales entourent de toutes parts les neurones. ${ }^{23}$ Dans les études au microscope électronique, cette hypothèse trouve un support dans le fait que la pénétration de substances liposolubles à travers la barrière hémato-encéphalique ressemble à la pénétration de ces mêmes substances à travers une membrane cellulaire et dans le fait que la barrière hémato-encéphalique fait défaut justement dans les régions où les capillaires ne sont pas couverts par les prolongements gliaux.

2) Torack et coll..$^{24}$ et Gerschenfeld et coll. ${ }^{25}$ montrent que dans l'œè̀me cérébral expérimental on constate un gonflement des cellules gliales sans distension correspondante d'un quelconque espace extracellulaire.

3) Katzman ${ }^{26}$ démontre que la composition électrolytique des cellules gliales, forte en $\mathrm{Na}^{+}$et en $\mathrm{Cl}^{-}$, ressemble plutôt à celle d'un liquide extracellulaire qu'à celle d'une liquide intracellulaire.

4) Les cellules gliales sont certainement métaboliquement actives, car Coggeshall et Fawcett ${ }^{27}$ ont trouvé dans le cytoplasme des cellules gliales de grandes quantités de glycogène et de nombreuses gouttelettes de graisse, c'est-à-dire tout le nécessaire pour produire l'énergie indispensable aux processus de transport.

5) D'après Kuffler et Potter ${ }^{28}$ il existe, parmi les cellules gliales, un passage intensif de courant électrique d'une cellule à l'autre. Donc une modification localisée est capable d'être transmise à une zône éloignée.

6) Hild et Tasaki ${ }^{29}$ ont montré in vitro que les cellules gliales manifestent de la pinocytose. De plus elles sont capables d'absorber des protéines ${ }^{30}$ et de le ferritine: ${ }^{31}$ elles sont donc capables de transporter des molécules relativement grandes.

L'hypothèse selon laquelle la glie constitue un espace extracellulaire fonctionnel, permettrait de donner une explication unitaire à la barrière hématoencéphalique.

Tout récemment ces différents problèmes ont été repris au cours du Symposium sur la barrière hémato-encéphalique, qui s'est tenu à Hilversum l'année dernière. $\mathrm{Ni}$ les apports biochimiques ni les apports anatomiques n'ont toutefois permis de conclure de façon définitive.

\section{Aspect endocrinologique}

Disons maintenant quelques mots sur notre troisième point de rappel de physiologie en envisageant l'aspect endocrinologique de la régulation du métabolisme de l'eau et des électrolytes dans le système nerveux central, sur lequel plus particulièrement des auteurs français ont mis l'accent. 
Ainsi que nous le lisons dans l'introduction du livre de Petit-Dutaillis et Bernard-Weil consacré au "Métabolisme de l'eau dans les Traumatismes du Crâne": ${ }^{32}$

La réalité biologique exige que l'on tienne compte, et le traumatisme cérébral l'exige plus impérieusement que tout autre, de l'action propre de l'hypophyse et de la corticosurrénale d'une part, de l'hormone post-hypophysaire de l'autre sur le métabolisme de l'eau. ... Les troubles du métabolisme de l'eau chez le traumatisé du crâne n'offrent pas que l'intérêt d'un trouble d'ordre général; leur étude est indissociable de celle des perturbations locales de la répartition et du volume de l'eau dans le cerveau et les espaces sous-arachnôidiens.

Ce rappel de physiologie nous permet de comprendre que des anomalies dans la répartition de l'eau et des électrolytes peuvent provoquer des troubles cliniques que nous allons passer en revue maintenant.

\section{ASPECTS CLINIQUES DES TROUBLES HYDRO-IONIQUES}

En clinique, les troubles hydro-ioniques peuvent être mis en évidence soit dans le sang, soit dans le liquide céphalo-rachidien, soit dans les urines, soit encore dans le tissu cérébral lui-même.

\section{Sang}

Les deux principaux syndromes observables dans le sang sont l'hyponatrémie et l'hypernatrémie. L'hyponatrémie est relativement fréquente dans les suites neurochirurgicales et est le plus souvent d'apparition précoce. Selon Linquette et coll. ${ }^{33}$ elle survient dans 18 pour cent des cas, mais sa fréquence est comparativement plus grande au cours des traumatismes graves (24\%), des tumeurs opérées (23\%) et des accidents neuro-vasculaires. L'hypertomie post-hypophysaire secondaire à l'agression provoque une augmentation de la volémie, qui tend à déterminer une hyponatrémie par dilution, puis une déperdition urinaire de sodium. Suivant les conditions d'hydration, deux possibilités se présentent: ou bien il s'installe une hyponatrémie avec hémodilution qui crée une intoxication par l'eau et un odème cérébral ou bien une hyponatrémie avec déshydratation qui peut conduire au collapsus cérébral. Toutefois la symptomatologie clinique est vague et ne nous permet pas d'affirmer ou d'infirmer des troubles de ce genre. Pour cela il nous faudra nous adresser aux examens de laboratoire.

Selon Bernard-Weil, la pathogénie de cet état se situe dans un déséquilibre entre l'axe antéhypophyso-surrénalien et la neuro-posthypophyse dans le sens d'une insuffisance cortico-surrénalienne ou d'une hyperactivité post-hypophysaire.

Cliniquement cet état peut entraîner un coma prolongé, une hémiplégie non régressive, une aphasie persistante et surtout une hypertension intracrânienne. Les crises d'épilepsie sont fréquentes au cours de ces syndromes.

Dans les suites neurochirurgicales, l'hypernatrémie est plus rare et tardive: toujours selon Linquette et coll., sa fréquence s'élève à 15 pour cent au cours des traumatismes graves et des tumeurs. Dans la plupart des cas l'hypernatrémie est secondaire à la déshydratation (par manque d'apport ou par excès d'élimination d'eau, comme dans le diabète insipide non corrigé). 
En clinique il existe également un syndrome, assez rare il est vrai, appelé "hypernatrémie neurogène," décrit par Cooper et Crevier en $1952 .^{34}$ Ce syndrome est caractérisé par un trouble de l'osmorégulation où se trouvent associés une adipsie et une hyperosmolarité plasmatique par rétention de chlorure de sodium sans signes cliniques de déshydratation, contrairement à l'hypernatrémie postopératoire. Il existe un amaigrissement important et on observe fréquemment un dérèglement neuro-végétatif, une élévation de l'azotémie et de la glycémie, une kaliémie normale et une insuffisance antéhypophysaire. Les manifestations comitiales sont rares. On note une diurèse faible et légèrement hypertonique ainsi que le maintien d'une natriurèse. Ces hypernatrémies neurogènes se rencontrent au cours de l'altération du lobe frontal à sa partie postérieure, de l'hypothalamus et du tronc cérébral. En 1966, Linquette et coll. ont émis l'hypothèse suivante pour expliquer la physio-pathologie de ce syndrome: ${ }^{35}$ un processus hypothalamique expansif serait responsable d'un syndrome polyuro-polydipsique latent, précédant ou non l'apparition d'une adipsie et provoquant une déshydratation progressive inapparente. Le maintien de la volémie s'expliquerait par une mécanisme osmolaire où l'hypernatrémie pourrait retenir l'eau même en l'absence d'hormone antidiurétique.

\section{Liquide céphalo-rachidien}

D'après Löwenthal, ${ }^{36}$ la concentration en potassium du liquide céphalorachidien augmente parallèlement à la concentration en protéines, ceci aussi bien dans les dissociations albumino-cytologiques que dans les hyperalbuminoses avec réaction céllulaire. Par contre dans l'atrophie cérébrale on note une diminution $\mathrm{du}$ potassium.

Il est intéressant de noter que l'ọ trouve une augmentation considérable de la teneur en sodium du liquide céphalo-rachidien chez les malades présentant une hypernatrémie dans le syndrome de Cooper et Crevier. ${ }^{34}$ Les méningites, par contre, sont fréquemment accompagnées d'une baisse de la concentration en sodium, de même que certains cas de dissociation albumino-cytologique.

\section{Urine}

Avec Löwenthal et coll..$^{37}$ nous avons étudié l'excrétion urinaire de sodium et de potassium dans une trentaine de cas neuro-chirurgicaux, comprenant 12 interventions sur la colonne rachidienne ou son contenu et 18 interventions intracrâniennes. Nous avons constaté une augmentation de l'élimination du potassium qui débute au cours des douze premières heures postopératoires. Cette élimination atteint jusqu’à 4 fois sa valeur normale, baisse ensuite jusqu’à revenir à cette valeur normale vers le $3^{\mathrm{e}}$ jour, puis continue à baisser, comme si l'organisme opérait à ce moment-là une rétention de potassium. Vers le $8^{\mathrm{e}}$ ou $10^{\mathrm{e}}$ jour cette élimination retourne progressivement à des valeurs normales.

Quant au sodium, c'est à une diminution de son élimination que nous assistons dès les premières heures après l'intervention. Elle atteint son minimum vers le $2^{\mathrm{e}}$ ou $3^{\mathrm{e}}$ jour, remonte ensuite mais reste en-dessous de la normale jusqu'au $8^{\mathrm{e}}$ jour.

Nous en avons conclu que pour le malade opéré la période critique au point 
de vue de la balance ionique commence vers le $3^{\mathrm{e}}$ jour postopératoire et se prolonge jusqu'au $8^{e}$ jour environ. De plus il semble que les interventions intracrâniennes atteignent plus profondément le métabolisme électrolytique que les interventions intrarachidiennes.

Certains ont voulu voir dans l'élimination de potassium une conséquence de la destruction tissulaire due à l'intervention chirurgicale. Toutefois de nombreux auteurs ont démontré que les pertes en azote ne sont pas parallèles aux pertes en potassium. Nous avons même observé une augmentation considérable de l'élimination du potassium dans des cas entraînant une destruction minime de substance tels que l'installation d'un drainage ventriculaire.

D'autres auteurs, tels que Gros, Minvielle et Vlahovitch, ont établi des courbes de teneurs sanguines en éosinophiles après des interventions neurochirurgicales. Elles sont remarquablement parallèles aux variations du rapport $\mathrm{Na} / \mathrm{K}$ que nous avons observées. Ceci semble donc plutôt appuyer l'hypothèse selon laquelle les modifications électrolytiques sont la conséquence du stress induit par un dérèglement postopératoire des secrétions internes.

\section{Tissu cérébral}

Les recherches biochimiques de Löwenthal ont démontré la constance de la composition électrolytique des diverses structures et régions du cerveau. Toutefois, après traumatisme ou intervention chirurgicale, il a souvent observé d'importantes modifications: une baisse de la teneur en potassium et une augmentation de la concentration en sodium. La substance blanche semble plus particulièrement atteinte par ces variations.

De même nous avons procédé à des déterminations de teneur en sodium et en potassium de tissus tumoraux et de tissus péritumoraux prélevés au cours d'interventions neurochirurgicales.

Comme matériel d'investigation nous avons disposé de 14 fragments de glioblastomes, de 5 fragments d'astrocytomes ainsi que de 11 fragments de tissu péritumoral et de 6 fragments de substance blanche œdématiée, tous vérifiés par examen histologique. Nous avons calculé la moyenne arithmétique des valeurs obtenues par les déterminations de matière sèche, des teneurs en $\mathrm{Na}$ dans la matière sèche $\left(\mathrm{Na}_{\text {sec }}\right)$ et dans la matière humide $\left(\mathrm{Na}_{\mathrm{hum}}\right)$ ainsi que des teneurs en $\mathbf{K}$ dans la matière sèche $\left(K_{\text {sec }}\right)$ et dans la matière humide ( $\left.K_{\text {hum }}\right)$. Ces résultats sont tous exprimés en grammes pour cent. Nous avons consigné tous ces résultats dans le Tableau I.

TABLEAU I

\begin{tabular}{|c|c|c|c|c|c|c|}
\hline & $\begin{array}{c}\text { Nombre } \\
\text { de cas }\end{array}$ & $\begin{array}{l}\text { Pour cent } \\
\text { de } \\
\text { matière } \\
\text { sèche }\end{array}$ & $\mathrm{Na}_{\text {sec }}$ & $\mathrm{Na}_{\text {hum }}$ & $K_{\text {seo }}$ & $\mathbf{K}_{\text {hum }}$ \\
\hline $\begin{array}{l}\text { Normal } \\
\text { Péritumoral } \\
\text { Glioblastome } \\
\text { Astrocytome } \\
\text { Oedème }\end{array}$ & $\begin{array}{r}7 \\
11 \\
14 \\
5 \\
6\end{array}$ & $\begin{array}{l}28,3 \\
20,8 \\
18,9 \\
18,9 \\
16,9\end{array}$ & $\begin{array}{l}0,62 \\
0,89 \\
1,04 \\
1,02 \\
1,70\end{array}$ & $\begin{array}{l}0,17 \\
0,17 \\
0,19 \\
0,18 \\
0,28\end{array}$ & $\begin{array}{l}0,87 \\
1,11 \\
1,21 \\
0,95 \\
0,94\end{array}$ & $\begin{array}{l}0,24 \\
0,22 \\
0,22 \\
0,21 \\
0,15\end{array}$ \\
\hline
\end{tabular}


Nous pouvons en tirer les conclusions suivantes:

1) Le pourcentage de matière sèche diminue dans le sens tissu normal-tissu péritumoral-tumeur-œdème, c'est-à-dire que de plus en plus d'espace est occupé par l'eau.

2) Parallèlement la teneur en $\mathrm{Na}_{\text {lum }}$ va en augmentant tandis que la teneur en $K_{\text {hum }}$ va en diminuant. Ceci démontre que dans l'œè̀me cérébral nous avons incontestablement une surcharge en $\mathrm{Na}$ aux dépens de la teneur en $\mathrm{K}$.

3) $\mathrm{Si}$ nous considérons les teneurs en $\mathrm{Na}$ et en $\mathrm{K}$ en fonction de la matière sèche, nous constatons que le $\mathrm{K}$ augmente au cours du passage du tissu normal au tissu péritumoral pour baisser ensuite, lorsque l'on passe au tissu tumoral et à l'œdème. Le $\mathrm{Na}_{\text {sec }}$, par contre, augmente de façon constante. Ces modifications non parallèles sont démonstratives de perturbations importantes du métabolisme de l'eau et des électrolytes.

Il faudra tenir compte de ces modifications lors de l'établissement du traitement tel que nous allons l'envisager maintenant.

\section{TratTEMENT}

Le traitement des désordres hydro-ioniques se basera sur une série d'explorations de laboratoire:

1) L'ionogramme plasmatique: les valeurs normales sont

pour le Na: 130 à $140 \mathrm{mEq} . / \mathrm{L}$.,

pour le K: 4 à $5,4 \mathrm{mEq} . / \mathrm{L}$.

pour le Cl: 95 à $105 \mathrm{mEq} . / \mathrm{L}$.

Les valeurs de $\mathrm{Na}$ et de $\mathrm{Cl}$ sont concordantes sauf s'il existe des perturbations acido-basiques: ainsi nous aurons de l'alcalose dans l'hypochlorémie et de l'acidose dans l'hyperchlorémie. Dans ces cas de déséquilibre il convient de mesurer la réserve alcaline et le $\mathrm{pH}$.

2) La protidémie et l'hématocrite: ceux-ci permettent d'évaluer le degré d'hémodilution ou d'hémoconcentration.

3) L'ionogramme globulaire sur lequel se base Bernard-Weil pour évaluer la teneur cellulaire globale en $\mathrm{K}$. La teneur globulaire normale en $\mathrm{K}$ est de 85 à $105 \mathrm{mEq} . / \mathrm{L}$.

4) Le volume de la diurèse, lionogramme urinaire et la densité urinaire: dans l'interprétation du volume de la diurèse c'est surtout la polyurie qui aura de la valeur, car l'oligurie accompagne souvent des pertes extra-rénales importantes.

5) L'élimination hormonale, comprenant: a) le dosage des 17 céto-stéroïdes et des 17 hydroxycorticoides, b) l'appréciation indirecte du facteur anti-diurétique par la mesure de la clearance à l'eau libre.

Ces mesures et dosages de laboratoire ne permettent toutefois pas d'éliminer l'observation clinique du malade. Disposant de ces données, nous pouvons entamer le traitement des désordres hydro-ioniques tel qu'il nous est proposé par le Professeur Laine et son équipe de la Clinique Neuro-Chirurgicale de Lille. ${ }^{38}$ Ce traitement visera avant tout à corriger les troubles de la natrémie et d'éventuels troubles de la kaliémie. Il devra également assurer les apports hydriques et caloriques indispensables. 
Troubles de la natrémie

Les deux grands troubles de la natrémie sont évidemment l'hyponatrémie et l'hypernatrémie. Toutefois nous rencontrerons également des états cliniques, graves ou bénins, où la natrémie sera parfaitement normale.

L'hyponatrémie peut être accompagnée d'une hémodilution ou d'une osmolarité normale ou même élevée. Dans l'hyponatrémie avec hémodilution la logique impose de réduire les apports d'eau et d'augmenter l'apport sodé. Etant donné leur propriété de favoriser la rétention sodée et d'augmenter l'excrétion d'eau, il y aura lieu d'administrer des corticoïdes à la dose de 100 à $150 \mathrm{mg}$. de cortisone deux fois par jour et de $40 \mathrm{mg}$. d'ACTH retard pendant la même période. Les solutions hypertoniques et les diurétiques contribueront à l'élimination de l'excès d'eau.

Dans l'hyponatrémie avec osmolarité normale ou élevée ce sera principalement la corticothérapie qui sera appliquée. Elle sera accompagnée d'une administration de sérum salé isotonique.

La physiopathologie de l'hypernatrémie attribue ce trouble à deux mécanismes principaux: hypernatrémie par manque d'eau (soit par apport insuffisant, soit par élimination excessive, comme dans le diabète insipide) ou hypernatrémie par insuffisance d'élimination d'eau.

Dans les cas de manque d'apport d'eau, le traitement qui s'impose est la réhydratation au moyen de glucose ou de Sorbitol isotonique à raison de 4 à 5 litres par jour. Cette réhydratation sera accompagnée d'un administration de 4 à $5 \mathrm{gm}$. de $\mathrm{KCl}$ par 24 heures et de deux fois 5 unités-retard d'extrait posthypophysaire.

Les cas d'hypernatrémie par défaut d'élimination de $\mathrm{Na}$ commandent surtout la surveillance du diabète insipide par l'extrait post-hypophysaire et par l'administration d'hexadécadrol, qui provoque une élimination de $\mathrm{Na}$, à raison de 4 à 8 mg. par jour.

Restent les cas où, malgré un état clinique perturbé, on ne trouve aucune modification de la natrémie: ainsi nous pouvons nous trouver en présence de traumatismes graves avec œdème cérébral, collapsus cérébral ou diabéte insipide sans que l'on puisse noter une perturbation de l'ionogramme.

Dans ces cas cliniques graves il sera bon d'instituer une corticothérapie et d'administrer des solutions hypertoniques et des diurétiques, tout en pratiquant régulièrement des dosages de sodium: si une hyponatrémie apparait, on adjoindra de l'ACTH au traitement; si, au contraire, c'est une hypernatrémie qui se dessine, on surveillera la diurèse et on donnera de l'extrait post-hypophysaire ou de l'hexadécadrol.

Dans les formes bénignes on se contentera d'assurer l'équilibre hydro-ionique et d'administrer des solutions hypertoniques.

\section{Troubles de la kaliémie}

Les deux indications majeures pour un apport de $\mathrm{K}$ sont d'une part les hypokaliémies (provoquées par hémodilution ou par pertes urinaires excessives entretenues par la corticothérapie), d'autre part les déshydratations cellulaires. Cet apport de $\mathrm{K}$ se fera par voie digestive ou intraveineuse à la dose de $3 \mathrm{gm}$. par 
jour ou plus. Le chlorure de potassium sera donné dans les cas avec alcalose, alors que le lactate, le citrate ou le gluconate de potassium seront administrés dans les cas accompagnés d'acidose. L'hyperkaliémie, elle, obéit au traitement de l'hémodilution.

\section{Apport calorique}

Un hypercatabolisme précède les perturbations hydro-ioniques, tout au moins dans les suites neurochirurgicales, et est à l’origine d'un déficit énergétique. Pour cette raison un apport calorique est indispensable chez ces malades.

Lazorthes et Campan estiment cet apport à 2.000 ou 2.500 calories par jour, se répartissant de la manière suivante:

ration de base: $1.500 \mathrm{cal}$., supplément par degré de fièvre: $200 \mathrm{cal}$., supplément en cas d'agitation: $300 \mathrm{cal}$., supplément pour compenser la destruction tissul.: $150 \mathrm{cal}$.

Au point de vue qualitatif, il convient de prévoir de 50 à $100 \mathrm{gm}$. de protéines par jour. Les besoins glucidiques seront couverts par l'administration intraveineuse de glucose hypertonique à 30 ou à 10 pour cent avec de l'insuline, qui s'avère avoir également une action anti-œdémateuse.

\section{Apport hydrique}

L'eau endogène métabolique émise au cours de cet hypercatabolisme postagressif doit être compensée par un apport hydrique. Cet apport sera établi en fonction de la diurèse et des déperditions cutanées et respiratoires. Il sera d'environ 1 litre à 1,5 litre dans les premiers jours et augmentera progressivement au fur et à mesure que le volume de la diurèse se normalise ou que les déperditions extra-urinaires augmenteront.

\section{CONCLUSION}

Au terme de ce travail nous aurions voulu arriver à établir des rapports plus étroits entre la physio-pathologie et la clinique, d'une part, et la thérapeutique, d'autre part. Cela est difficile car les moyens d'investigation au lit du malade sont limités et, de plus, les observations faites par anatomistes et physiologistes ne permettent pas encore d'aboutir à des conclusions concordantes, qui nous permettraient de sortir enfin du domaine de l'hypothèse.

Les conclusions du Workshop on Brain Edema de Vienne $1965^{30}$ et du Symposium sur la barrière hémato-encéphalique d'Hilversum $1966^{15}$ nous ont démontré que les connaissances sur l'espace extracellulaire et sur l'œdème cérébral ont fait d'incontestables progrès. Toutefois on ne peut s'empêcher de regretter que ces résultats, qui sont l'aboutissement de recherches faites avec un luxe de moyens et une précision scientifique extraordinaires, ne permettent pas encore d'en tirer des conclusions cliniques définitives.

Il faudra donc momentanément encore se contenter, au lit du malade, des modestes moyens d'investigation dont nous disposons actuellement. L'observation clinique nous permet de dire à quel moment il faut avoir recours aux examens 
de laboratoire et quel genre de dosage devra être pratiqué. Le pronostic dépendra essentiellement des résultats obtenus.

Si ces conclusions peuvent sembler quelque peu décevantes et désabusées, nous devons nous consoler en pensant que grâce à cette thérapeutique nous avons quand même souvent pu corriger des troubles hydro-ioniques postopératoires.

\section{Summary}

At the end of this paper, we would like to have succeeded in establishing some closer relations between the pathophysiological and clinical aspects on the one hand and the therapeutic on the other. That is difficult because the means of investigation at the patient's bedside are limited and, moreover, the observations done by anatomists and physiologists do not allow us to come to more than hypothetical conclusions.

The conclusions of the "Workshop on Brain Oedema" at Vienna, 1965, and of the Symposium on "Hemato-Encephalic Barrier" at Helversum, 1966, have shown that knowledge of the extracellular space and of cerebral oedema has made real progress. Otherwise, we can only regret that those results, obtained by research done by the most expensive means and with an extraordinary scientific precision, do not permit one to draw definite clinical conclusions.

Consequently, we will still have to work at the bedside for a while with the poor means of investigation that we actually have. Clinical observation allows us to say when one must ask for laboratory tests and what sort of dosage should be given. Prognosis will depend necessarily on the results obtained.

If such conclusions may seem a bit deceiving and discouraging, we must be stimulated by knowing that, thanks to such a therapeutic approach, we have often been able to treat postoperative hydro-ionic disorders successfully.

\section{REMERCIEMENTS}

Remerciements au Docteur P. Selosse pour le prélèvement soigneux des pièces anatomiques en cours d'intervention, au Docteur L. van Bogaert et au laboratoire de Neuropathologie de la Fondation Born-Bunge pour l'établissement des diagnostics histologiques, ainsi qu'au Docteur D. Karcher et à Madame G. Roelants pour l'exécution des dosages biochimiques.

\section{REFERENCES}

1. Grundfest, H. The Nature of the Electrochemical Potentials of Bioelectric Tissues. Electrochemistry in Biology and Medicine, T. Shedlovsky, ed. New York: John Wiley (1955) pp. 141-66.

2. Honckin, A. L. Ionic Movements and Electrical Activity in Giant Nerve Fibres. Proc. Roy. Soc. B. 148: 1 (1958).

3. -- The Ionic Basis of Electrical Activity in Nerve and Muscle. Biol. Rev. 26: 339 (1951).

4. Eccles, J. C. The Physiology of Nerve Cells. Baltimore: Johns Hopkins Press (1957).

5. Fernandez-Moran, H. Cell-membrane Ultrastructure. Symposium on the Plasma Membrane. New York: Amer. Heart Ass, and N.Y. Heart Ass. (1962), p. 1039. 
6. Kety, S. Circulation and Metabolism of the Human Brain in Health and Disease. Amer. J. Med. 8: 205 (1950).

7. Mangold, R.; Sokoloff, L.; Conneh, E.; Kleinerman, J.; Therman, P. O.; \& Kety, S. S. The Effects of Sleep and Lack of Sleep on the Cerebral Circulation and Metabolism of Young Men. J. Clin. Invest. 34: 1092 (1955).

8. Soroloff, L.; Mangold, R.; Wechsler, R. L.; Kenney, C.; \& Kety, S. S. The Effect of Mental Arithmetic on Cerebral Circulation and Metabolism. J. Clin. Invest. 34: 1101 (1955).

9. Schaltenonand, G. \& Banley, P. Die perivaskuläre Piagliamembran des Gehirns. J. Psychol. Neurol. 35: 199 (1929).

10. RoBin, E. D. Some Aspects of Fluid and Electrolyte Metabolism in the Brain. Anesthesiology. 26: 791 (1965).

11. Reed, D. J. \& Woodbury, D. M. Kinetics of C14 Sucrose Distribution in Cerebral Cortex, Cerebrospinal Fluid, and Plasma of Rats. Fed. Proc. 19: 80 (1960).

12. Barlow, C. F.; Domek, N. S.; Goldberg, M. A.; \& Roth, L. J. Extracellular Brain Space Measured by $S^{35}$ Sulfate. Arch. Neurol. 5: 102 (1961).

13. Kibler, R. F.; O'Neml, R. P.; \& Robin, E. D. Intracellular Acid-base Relations of Dog Brain with Reference to the Brain Extracellular Volume. J. Clin. Invest. 43: 431 (1964).

14. Rall, D. P. \& Patlak, C. S. Extracellular Space in the Brain. Fed. Proc. 21: 324 (1962).

15. Symposium sur la barrière hémato-encéphalique. Hilversum, 1966 (en préparation).

16. Rali, D. P. The Structure and Function of the Cerebrospinal Fluid. In The Cellular Functions of Membrane Transport, J. F. Hoffman, ed. New York: Prentice-Hall (1964), p. 269.

17. Felderer, N. \& Fleischinuer, K. A new experimental approach to the physiology and pharmacology of the brain. Brit. Med. Bull. 21/1: 36-43, 1965.

18. Dempsey, E. W. \& Wistocki, G. B. An Electron Microscopic Study of the Blood-Brain Barrier in the Rat, Employing Silver Nitrate as a Vital Stain. J. Biophys. Biochem. Cytol. 1: 245 ( 1955 ).

19. Luse, A. Electron Microscopic Observations of the Central Nervous System. J. Biophys. Biochem. Cytol. 2: 531 (1956).

20. Wyckoff, R. W. G. \& Young, J. Z. The Motorneuron Surface. Proc. Roy. Soc. (Biol.) 144: 440 (1956).

21. De Robertis, E. Submicroscopic Morphology of the Synapse. Int. Rev. Cytol, 8: 61 (1959).

22. Lumsden, C. Functional Aspects of the Glial Apparatus. Acta Neurol. Belg. 57: 472 (1957).

23. De Robertis, E. \& Gerschenfeld, H. M. Submicroscopic Morphology and Function of Glial Cells. Int. Rev. Neurobiol. 3: I (1961).

24. Torack, R. M.; Terry, R. D.; \& Zimmerman, H. H. The Fine Structure of Cerebral Fluid Accumulation: I. Swelling Secondary to Cold Injury. Am. J. Pathol. 35: 1135 (1959).

25. Gerschenfeld, H. M.; Tramezzani, J. H.; \& De Robertis, E. Ultra-structure and Function in Neurohypophysis of the Toad. Endocrinology, 66: 741 (1960).

26. Katzman, R. Electrolyte Distribution in Mammalian Central Nervous System: Are Glia High Sodium Cells? Neurology, 11: 27 (1961).

27. Coggeshall, R. E. \& Fawcetr, D. W. The Fine Structure of the Central Nervous System of the Leech, Hirudo Medicinalis, J. Neurophysiol. 27: 229 (1964).

28. Kuffler, S. W. \& Potter, D. D. Glia in the Leech Central Nervous System: Physiological Properties and Neuron-glia Relationship. J. Neurophysiol. 27: 290 (1964).

29. Hind, W. \& TASAkI, I. Morphological and Physiological Properties of Neurons and Glial Cells in Tissue Culture. J. Neurophysiol. 25: 277 (1962).

30. Klatzo, I. \& Miguel, J. Observations on Pinocytosis in Nervous Tissue. J. Neuropath. Exper. Neurol. 19: 457 (1960).

31. Rosenbluth, J. \& Wissig, S. The Uptake of Ferritin by Toad Spinal Ganglion Cells. J. Cell. Biol. 19: 19A (1963).

32. Petit-Dutaillis, D. \& Bernard-Weil, E. Métabolisme de l'eau dans les traumatismes du crâne. Paris: Masson (1958).

33. Linquette, M.; Laine, E.; Fossati, P.; Lefebvne, J.; \& Racadot, N. Les Syndromes d'hyponatrémie et d'hypernatrémie en neurochirurgie: Essai de physio-pathologie 
neuro-endocrinienne: Conséquences thérapeutiques. Rev. français endocrinol. clin. 6: 7 (1965).

34. Cooper, J. S. \& Crevier, P. H. Neurogenic Hypernatremia and Hyperchloremia. J. Clin. Endocrinol. 12: 821 (1952).

35. Linquette, M.; Laine, E.; Fossati, P.; Delantsheer, J. M.; Lefebvre, J.; Racadot, N.; \& Christiaens, J. L. Les Hypernatrémies neurogènes. Lille médical. 11: 950 (1966).

36. Löwenthat, A. Composition du cerveau en certains éléments minéraux et essai sur leur signification en pathologie. Acta Med. Belg. (1958).

37. Löwenthal, A.; Soetens, A.; \& Van Sande, M. Variations de l'excrétion urinaire des ions $\mathrm{K}$ et $\mathrm{Na}$ dans les suites des interventions neuro-chirurgicales. Rev. Belge de Path. \& de Méd. Exp. 26: 112 (1957).

38. Laine, E.; Fossati, P.; \& Lefebvne, J. Traitement des désordres hydro-ioniques observés en neurochirurgie. Problèmes de Réanimation (Joumées de Réanimation MédicoChirurgicale, Nancy, 1964) pp. 463-519.

39. Workshop on Brain Edema. Vienne, 1965 (en préparation). 